\title{
What dot clusters and bar graphs reveal: Subitizing is fast counting and subtraction
}

\author{
David B. Boles, Jeffrey B. Phillips, And Somer M. Givens \\ University of Alabama, Tuscaloosa, Alabama
}

\begin{abstract}
In two studies, we found that dot enumeration tasks resulted in shallow-sloped response time (RT) functions for displays of 1-4 dots and steep-sloped functions for displays of 5-8 dots, replicating results implicating subitizing and counting processes for low and high ranges of dots, respectively. Extracting number from a specific type of bar graph within the same numerical range produced a shallow-sloped but scallop-shaped RT function. Factor analysis confirmed two independent subranges for dots, but all bar graph values defined a unitary factor. Significantly, factor scores and asymmetries both showed correlations of bar graph recognition to dot subitizing but not to dot counting, strongly suggesting that subitizing was used in both enumeration of low numbers of dots and bar graph recognition. According to these results, subitizing appears to be a nonverbal process operating flexibly in either additive or subtractive fashion on analog quantities having spatial extent, a conclusion consistent with a fast-counting model of subitizing but not with other models of the subitizing process.
\end{abstract}

There is evidence that the same perceptual process can be involved in both dot enumeration and bar graph recognition involving judging the numerical quantities represented by small dot clusters and by bar graphs. Taking a factoranalytic approach and using limited-time (100-msec) displays, Boles (1991, Experiment 1) asked participants to make lateralized odd-even judgments of clusters of 1-8 dots, as well as of a specific kind of bar graph that showed whole number values from 1-8 (see Figure 1). Several other lateralized tasks were used as well.

Both numerical tasks showed robust, highly significant lateral differences favoring the left visual field (LVF), or right hemisphere. More importantly, when all the lateral differences were subjected to factor analysis, the visual dots and bar graph scores correlated +.47 and +.71 , respectively, to the same factor. In contrast, a third task involving odd-even judgments of numbers expressed as words (e.g., "one") showed a robust RVF (left hemisphere) lateral difference that loaded on a separate factor. On the basis of these results, a spatial quantitative process common to the dots and bar graph tasks and predominantly lateralized in most right-handed people to the right hemisphere was deemed to have been identified. The independence of this process from a number of others, including others lateralized to the right hemisphere, has since been amply confirmed (Boles, 1992, 1996, 1998, 2002).

Another approach looked at transfer of training between numerical tasks (Boles, 1997). Three groups of participants were first given the bar graph recognition task to establish a baseline of performance; they then variously received two task repetitions of bar graph recognition, dot enumeration, or recognition of numbers expressed as words. These tasks were followed by another iteration of the bar graph recognition task. The questions were whether intervening practice on the dot enumeration task would improve performance on the bar graph task between the first and the last administration and, if so, how the performance improvement would compare with that of the groups that received intervening bar graphs or words.

The somewhat startling outcome was that intervening dot enumeration improved response time (RT) performance almost as much as intervening bar graph recognition itself and far more than intervening word recognition. Because the three tasks were identical in decision processes (oddeven judgment) and responses (manual two-choice RT), the results strongly implied that dot enumeration and bar graph recognition shared a common perceptual process not shared by word recognition.

A final approach to the question of commonality has been to examine the effectiveness of time-sharing dot enumeration and bar graph recognition. If a common process is involved, the tasks should not time-share well in comparison with pairings of tasks that have less in common. Boles and Law (1998, Experiment 1) confirmed this expectation within a broader program of testing hemispheric resource theories, finding strong evidence of selective interference between dots and bar graphs. Thus, when bar graph recognition was performed simultaneously with dot enumeration, bar graph errors were nearly five times greater than when bar graph recognition was performed simultaneously with a color-naming task. The opposite was true of a word recognition task: More errors were made when word recognition was paired with color-naming than with dot enumeration tasks.

D. B. Boles, dboles@bama.ua.edu 


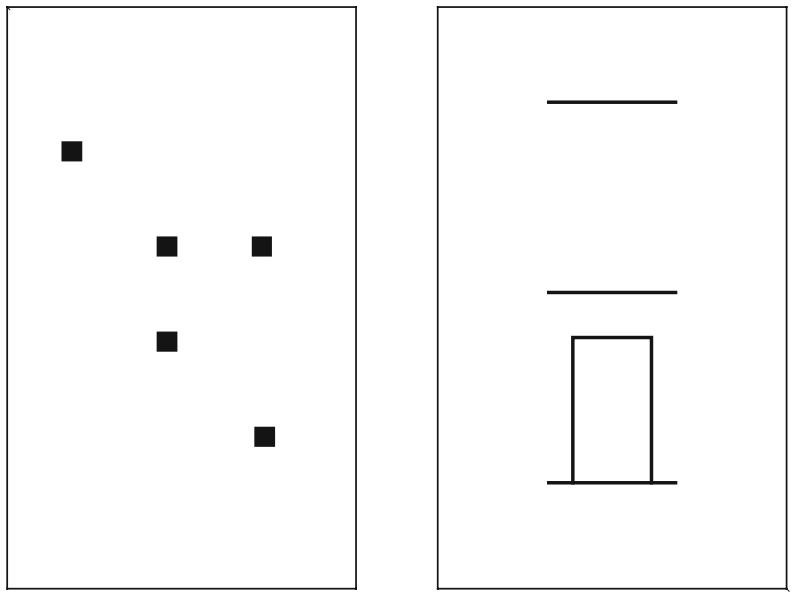

Figure 1. Examples of dot cluster and bar graph stimuli, representing the numbers 5 and 3 , respectively.

Thus, empirical results from a variety of approaches strongly converge on the conclusion that dot enumeration and bar graph recognition can involve a common process. That process appears to be perceptual in nature, given the experimental design in the transfer-of-training study. Furthermore, given the visual field (VF) results, the common process is predominantly lateralized to the right hemisphere of the brain.

However, if one steps beyond the empirical results and asks specifically what process the two tasks have in common, answers do not seem to be quite as forthcoming. It is one thing to infer a spatial quantitative process from a common factor loading of task measures, but it is another thing to describe it. What kind of process can work on both (1) stimuli that are point-like and express magnitude on the basis of numerosity (i.e., dots), and (2) stimuli that are box-like and express magnitude on the basis of location of one side relative to external landmarks (i.e., bar graphs)? What seems the obvious answer-that the two tasks are generally numerical-is certainly incorrect because, as the factor-analytic and transfer-of-training studies showed, a separate process is involved in recognizing numbers expressed as words, and it is lateralized predominantly to the left hemisphere (Boles, 1991, 1992, 1996, 1998, 2002).

The commonality mystery deepens when one considers that dot enumeration involves several different perceptual processes. A well-developed literature reports studies of judgments of the numerosity of dots ranging from 1 dot upward, not merely truncated at 8 dots as in the studies described above. Mandler and Shebo (1982) reviewed the early work and concluded that a characteristic set of results was obtained when display time was limited - to, for example, a 200-msec exposure. Specifically, there was an initial shallow-sloped function relating RT to dot numerosity for up to approximately 4 dots, followed by a steepsloped function up to about 6 dots, after which the slope flattened (see, e.g., Kaufman, Lord, Reese, \& Volkmann, 1949; Mandler \& Shebo, 1982). Others have reported that the flattening occurs at slightly higher numerosities - that is, with 7 or 8 dots (Dehaene \& Cohen, 1994; Sathian et al., 1999; Wender \& Rothkegel, 2000).

These results indicate that dot enumeration does not involve a unitary perceptual process, even if the task is limited to 8 dots, because three separate functions relate RT to numerosity: The first is a shallow-sloped function for up to 4 dots; the second, a steeply sloped function for up to 6-8 dots; and the third, a flat function for more than 6-8 dots. Separate processes have been attributed to each of the three functions, labeled subitizing, counting, and estimation, respectively.

\section{Subitizing}

The term subitizing has come to be applied specifically to the process presumed to underlie the early portion of the numerosity curve, the one involving a small number of dots and yielding a shallow slope. Although Mandler and Shebo (1982) suggested that the use of subitizing extends upward to 4 dots, the limit varies somewhat from study to study. In children, for example, subitizing may extend upward only to 3 dots (Chi \& Klahr, 1975; Svenson \& Sjöberg, 1978).

But what exactly is the subitizing process? A rich array of hypotheses has been put forward. Two early hypotheses described by Averbach (1963) held that the rapid processing of small numbers of items is accounted for either by (1) Weber's law, based on the fact that an integer difference between adjoining numbers is a larger proportion of the number to be recognized when that number is smaller than when it is larger; or (2) limits in short-term memory, such that storage limits constrain position counting less with smaller than with larger numbers. Mandler and Shebo (1982), on the other hand, proposed that fast processing of small numbers resulted from learning canonical patterns: One dot is a dot, but two dots are a line, and three dots, a triangle. Thus, by perceptually completing and recognizing the geometric form, we might rapidly extract number. A related view was recently expressed by Logan and Zbrodoff (2003), who proposed that the greater visual similarity of low-numerosity displays, rather than canonical form, supports fast pattern matching in the subitizing range.

Gallistel and Gelman (1992), however, considered subitizing to be a preverbal counting process shared by human and nonhuman species alike. This fast-counting model holds that each element in a pattern increases a mental magnitude by one count, very rapidly. Although their model was based on animal studies using sequences rather than simultaneously presented groups of stimuli, Gallistel and Gelman believed that it was generalizable to analog quantities, including (interestingly) histograms or bar graphs. A limit on fast counting was proposed to occur with numerosities greater than four or five, because of accumulated variability in the magnitude produced, resulting in increased errors with larger numbers. Above that limit, verbal counting was proposed to be used, at least by human enumerators.

In their FINST model, Trick and Pylyshyn $(1993,1994)$ viewed subitizing as a preattentive but limited-capacity tagging of item locations. Their idea was that when confronted with an array of objects, the observer initially determines the objects' locations as a prelude to addi- 
tional processing, including object recognition. Although locational tagging was viewed as preattentive, Trick and Pylyshyn proposed that only a limited number of item locations could be tagged, so that once the subitizing range was surpassed, counting was used, with attention moving from one part of a display to another.

Although other researchers have continued to investigate the subitizing phenomenon, they have generally done so within the described frameworks or within closely related ones (Basak \& Verhaeghen, 2003; Fink et al., 2001; Sathian et al., 1999; van Oeffelen \& Vos, 1982; Watson \& Maylor, 2006; Wender \& Rothkegel, 2000). Dehaene and Cohen (1994), for example, reported results that they viewed as consistent with a parallel procedure conducted on small numbers of items, similar in conception to the FINST model.

Several of these studies, including Dehaene and Cohen's (1994), have attempted to answer not only cognitive questions about subitizing, but neurally based questions as well. Sathian et al. (1999) and Fink et al. (2001) assessed the localization of subitizing using PET and fMRI, respectively, and found significant activation in left and right occipital cortex (as well as in several areas specific to one but not both studies). Although the occipital activation was apparently unlateralized in the fMRI study, there was some indication of greater right hemisphere involvement in the PET study. However, a role of the inferior parietal cortex in subitizing is also possible, since activation was found there in some comparisons in Fink et al. and also in a recent event-related potential study (Nan, Knösche, \& Luo, 2006). An inferior parietal locus may also be consistent with task asymmetry intercorrelations (Boles, 2002).

\section{Counting}

In contrast to the fast subitizing process used for small numerosities, for arrays of 4 or 5 items up to 7 or 8 items, a slower counting process is thought to be used. Such a process is implied by the steeper slopes of the numerosity curves within this range, perhaps $150-370 \mathrm{msec}$ per item, as opposed to $10-100 \mathrm{msec}$ per item within the subitizing range. The interpretation of a steeper slope as implicating a counting process appears uncontroversial (Dehaene \& Cohen, 1994; Mandler \& Shebo, 1982; Oyama, Kikuchi, \& Ichihara, 1981; Sathian et al., 1999; Trick \& Pylyshyn, 1993, 1994; Wender \& Rothkegel, 2000). The counting process makes demands on working memory, and on that basis substantial differences among individuals emerge. Individuals with low verbal-memory spans perform more slowly within the counting range than do individuals with high spans, whereas they do not differ in subitizing (Tuholski, Engle, \& Baylis, 2001).

An attempt to localize the site of processing for the counting portion of the numerosity curve has yielded somewhat unclear results. In their PET study, Sathian et al. (1999) found activation at multiple sites, primarily but not exclusively in the right hemisphere.

\section{Estimation}

At some point between 6 and 8 items in a display, the numerosity curve again begins to flatten, thenceforth in- creasing with a slope of only about $10-50 \mathrm{msec}$ per item (Mandler \& Shebo, 1982). In this range, assuming that display duration is limited, a counting strategy is no longer possible and estimation is believed to be used instead (Kaufman et al., 1949). Oyama et al. (1981) used the term guessing for the same process. However, the term estimation more accurately captures the observation that as numerosity increases, numerical responses also increase, even though error does as well.

These results indicate that the dot enumeration task involves not one but three separate enumeration processes. Such a multiplicity of processes further deepens the mystery surrounding what process dot enumeration and bar graph recognition could have in common, although a common process is strongly implied by common factor loadings, successful transfer of training, and selective interference. On the surface, it is not obvious that the recognition of bar graphs might involve subitizing (the rapid and confident enumeration of small collections of items), counting (the time- and attention-consuming incrementing of numerical quantity), or estimation. A bar graph such as the one shown in Figure 1 is a unitary stimulus, not a small collection of items; it has multiple reference points that would seem to make any straightforward counting strategy unlikely; and, at least when it requires odd and even responses, it demands a precise determination of quantity that would seem to make estimation unlikely.

Fortunately, the three-limbed numerosity curve provides a means of determining which process the bar graph recognition and dot enumeration tasks have in common. If performance is separately measured for each limb in the dot enumeration task and correlated across individuals to performance on the bar graph task, a positive correlation should be found if the process underlying a limb is also used in bar graph recognition. A positive correlation may also be found between the corresponding VF asymmetries.

To address this question, two studies were conducted. In the first, the data from prior experiments in our laboratory were aggregated to provide a large-sample investigation of correlations between bar graph and dot enumeration measures. On the basis of these results, a second, smaller study was conducted prospectively to provide confirmation of the major outcomes.

It should be noted that the focus of the studies was on the RT variable rather than on errors, for three reasons. First, significant asymmetries in the dot enumeration and bar graph recognition tasks are usually found only in RT (Boles, 1991, 1992, 1996, 2002), and the question of which hemispherically lateralized process the two tasks have in common is fundamental to the investigation. Second, the common factor loading of the dot cluster and bar graph asymmetries that raised the issue of a shared process was itself based on RT measures, not on error measures (Boles, 1991). Third, in the subitizing literature, the use of slopes in parsing the subitizing, counting, and estimation components has depended on RT. For these reasons, the inferential analyses employ RT, although brief descriptive statistics are provided for the error data as well.

By way of preview, it appears that a fortuitous aspect of the design of the bar graphs used in the previous 
research-unlabeled horizontal reference lines at the 0 , 4 , and 8 levels - induces the use of the subitizing process and that this design element accounts for the commonalities between bar graph and dot cluster processing. In turn, the commonalities are highly revealing of the nature of the subitizing process.

\section{STUDY 1}

\section{Method}

Participants. The aggregate sample for Study 1 initially consisted of data from 187 young adults who took part in previous factor-analytic experiments involving the bar graph recognition and dot enumeration tasks. These included 70 participants from Boles's (1991) Experiment 1 and 57 and 60 participants from Boles's (2002) Experiments 1 and 2, respectively. The final sample included data from 153 participants, following screening to ascertain that each participant (1) had a minimum of $60 \%$ correct responses on each of the two tasks, a criterion consistently maintained to make certain that RTs were interpretable when the chance response level was 50\% (see, e.g., Boles, 1991, 2002), and (2) had given correct responses for each numerical value in each task. All participants were right-handed by self-classification, and all completed the Edinburgh Handedness Inventory (Oldfield, 1971), which confirmed their right-handedness.

Apparatus and Stimuli. Stimuli were presented in a blue color on a dark background, using a monitor driven by an Apple IIe computer and Apple-Psych experimental software (Osgood, 1988). An illustration of the dot cluster and bar graph stimuli appears in Figure 1, although with dark figures on light backgrounds rather than vice versa. A dot cluster contained from 1-8 square dots (Figure 1) placed at random locations in an invisible $3 \times 4$ grid, with the grid subtending $4.1^{\circ}$ by $6.8^{\circ}$. The eccentricity for the dots task, from fixation to the grid's near edge, was $2.4^{\circ}$. A bar graph with a whole number value of 1-8 was plotted as a vertical rectangle with horizontal reference lines at the 0,4 , and 8 levels. These lines subtended a visual angle of $2.7^{\circ} \times 7.9^{\circ}$, with eccentricity from fixation to the near edge of $2.6^{\circ}$. For both tasks, bilateral displays were used, consisting of two stimuli selected at random, with an arrowhead ("<" or " >") at fixation indicating the stimulus to which the participant was to respond. Bilateral displays were used because they have been found to produce larger, more significant, and more reliable asymmetries than unilateral displays (Boles, 1983, 1987, 1990, 1995; Olk \& Hartje, 2001; Rayman \& Zaidel, 1991). An external keyboard was used to collect responses.

Procedure. The three experiments from which participant data were taken were slightly different procedurally, but all used a number of tasks in addition to dot enumeration and bar graph recognition. The additional tasks are irrelevant for present purposes and will not be discussed further, although Boles $(1991,2002)$ may be consulted for information concerning them. Task presentation order was counterbalanced across participants using a Latin square design in such a manner that dot enumeration and bar graph recognition were approximately equally often the first of the two tasks.

For both tasks, a trial involved a 750-msec fixation cross in central vision, followed by a $100-\mathrm{msec}$ blank period and then a display of the stimulus for $100 \mathrm{msec}$. Instructed to respond both as quickly and as accurately as possible, the participant then pressed one of two keys to indicate an odd $(1,3,5$, or 7$)$ or even $(2,4,6$, or 8$)$ response. The two keys were placed in the midline, with one key located closer to and one further away from the participant (i.e., there was no left-right arrangement). The participant placed a finger from each hand on each key, with hand assignments balanced across participants. After the participant responded or $4 \mathrm{sec}$ had elapsed, feedback was given. If the response was correct, the RT was displayed on the screen; if the response was incorrect or if $4 \mathrm{sec}$ had elapsed without a response, "Error" was displayed on the screen.
In both tasks, 24 practice trials were followed by 144 experimental trials, the latter split into blocks of 48 with the opportunity for brief rests in between.

\section{Results}

Correct responses were analyzed by calculating median RTs and percentage of errors for each number represented (1-8), separately in the LVF and RVF and separately for each task.

RT data. The RT data are illustrated in Figure 2. A repeated measures ANOVA using the factors of VF (LVF vs. RVF), number (1-8), and task (dot enumeration vs. bar graph recognition) found all three main effects to be significant $\left[F(1,152)=62.14, M S_{\mathrm{e}}=27,206, p<.001\right.$, for VF; $F(7,1064)=277.11, M S_{\mathrm{e}}=79,965, p<.001$, for number; and $F(1,152)=238.29, M S_{\mathrm{e}}=615,383, p<$ .001 , for task]. The VF $\times$ number interaction was also significant $\left[F(7,1064)=2.06, M S_{\mathrm{e}}=20,055, p<.05\right]$. As Figure 2 indicates, LVF means were smaller than RVF means across the full range of numbers (1-8) for both tasks, and the interaction indicates some differences were larger than others. However, since there was no VF $\times$ number $\times$ task interaction $\left[F(7,1064)=1.24, M S_{\mathrm{e}}=\right.$ $21,288]$, the effect is not discussed further.

The only other significant term was the number $\times$ task interaction $\left[F(7,1064)=233.36, M S_{\mathrm{e}}=85,517, p<\right.$ $.001]$. Visual inspection of Figure 2 suggests that for dot enumeration, there is a shallow-sloped subitizing range for represented numbers from 1 to about 4 , followed by a steep-sloped counting range for numbers from about 4 to 7. The lack of an RT increase from numbers 7 to 8 may indicate estimation, although this is hard to determine with only one value. Bar graphs, by contrast, show a very intriguing scallop-shaped function for numbers $1-3$, again for numbers 4-7, and perhaps starting again with number 8 ; this function suggests a shallow slope similar to that for the subitizing range.

Because of the relatively large data set available, it was possible to develop statistically based descriptions of these functions by employing factor analysis. The aim was first to reduce the data to an appropriate number of processing components in each task, then to determine

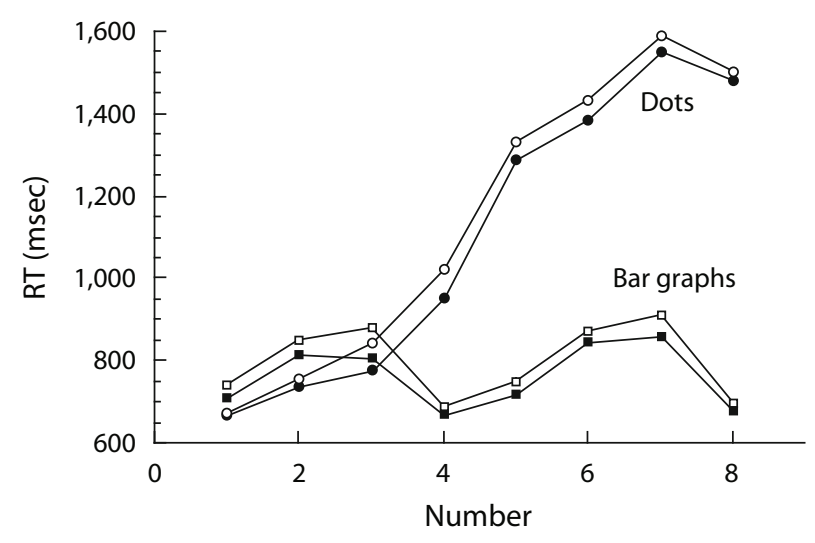

Figure 2. Response times from Study 1 . The closed symbols represent the left visual field; open symbols, the right visual field. 
relationships among the components across tasks. Initially, the RT data were collapsed across the VF variable, resulting in eight variables per task. With 153 participants and eight variables per task, the design more than satisfied the oft-cited recommendation that there be at least 5 participants per variable for a satisfactory factor analysis (Gorsuch, 1983).

For the dot enumeration task, principal-components analysis extracted two factors with eigenvalues exceeding unity (5.10 and 1.66 , with the third eigenvalue at 0.38 ), which together accounted for $85 \%$ of the variance. Because separate subitizing, counting, and estimation processes have been identified in previously published dot enumeration studies, we assumed that these processes should be orthogonal in factor analysis and thus selected the orthogonal varimax rotation procedure.

The procedure converged in three iterations, producing the rotated factor matrix loadings shown in Table 1. Clearly, there is strong evidence for a subitizing factor in the low-dots range and a counting factor in the high-dots range, with displays of 4 and 5 dots involving a mixture of both. There is no evidence in these results that 8 dots involved a separate estimation process.

For the bar graphs, principal-components analysis extracted only a single factor, with an eigenvalue of 5.99 (with the next eigenvalue at 0.57 ), which accounted for $75 \%$ of the variance. With only one factor, there is no rotation. All factor matrix loadings ranged between .84 and .89 . This result strongly supports the conclusion that a single enumeration process underlies the bar graph recognition results.

But which process? Having reduced the data to two dot enumeration components and one bar graph recognition component, the next step was to calculate the correlations between factor scores. Factor scores were calculated from the dot enumeration factor analysis, orthogonally representing the subitizing factor (Factor 2) and the counting factor (Factor 1). These were then correlated to the single bar graph factor score across participants. The results were unequivocal: The bar graph factor score was significantly correlated to the dot subitizing factor score $[r(151)=+.47, p<.001]$ but not to the counting factor score $[r(151)=+.06]$.

As the ANOVA results imply, similar LVF advantages were obtained for bar graphs $(33 \mathrm{msec}$ faster than results for the RVF) and for dot stimuli within the subitizing range (39 msec faster) and the counting range (40 msec faster). However, this does not necessarily indicate that the same

Table 1

Rotated Factor Matrix Loadings From Study 1, With All Loadings Greater Than .40 Shown

\begin{tabular}{ccc}
\multicolumn{3}{c}{ Loadings Greater Than .40 Shown } \\
\hline Variable & Factor 1 & Factor 2 \\
\hline 1 dot & & .87 \\
2 dots & & .90 \\
3 dots & .59 & .87 \\
4 dots & .83 & .64 \\
5 dots & .93 & .41 \\
6 dots & .93 & \\
7 dots & .93 & \\
8 dots & & \\
\hline
\end{tabular}

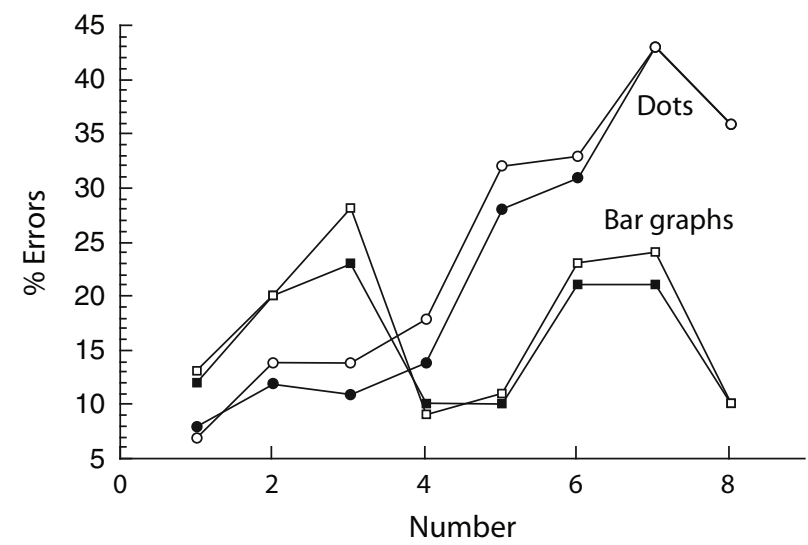

Figure 3. Percentage errors from Study 1 . The closed symbols represent the left visual field; open symbols, the right visual field.

right-hemisphere process is used by all three, since samehemisphere asymmetries often dissociate in correlation and in subsequent factor analysis, indicating the existence of separate processes (Boles, 1991, 1992, 1996, 2002). To examine this issue, VF differences were correlated across participants, using the same ranges of numerical values identified in the factor analysis. A significant correlation was found between asymmetry in the dot subitizing range and asymmetry in bar graph recognition $[r(151)=+.20$, $p<.02]$. Asymmetry in the dot counting range did not correlate to bar graph asymmetry $[r(151)=.00]$, and dot subitizing and dot counting asymmetries likewise did not correlate $[r(151)=+.11]$.

Percentage errors. The percentage error data are illustrated in Figure 3. Although noisier, they appear similar in form to the RT data, and there appears to be little in them to contradict the major conclusions from the RT data.

\section{Discussion}

Study 1 produced multiple sources of converging evidence that dot enumeration within the subitizing range and bar graph recognition share a common process, whereas dot enumeration within the counting range uses a separate process. Bar graph recognition and dot enumeration in the subitizing range show similar shallow-sloped functions relating numerical value to RT; for bar graphs, this function recurs over the 1-3 and 4-7 ranges. These slopes sharply contrast with the steep-sloped function underlying dot counting. Factor analysis performed separately on dot enumeration and bar graph recognition found two factors underlying dot subitizing and dot counting as well as a unitary factor underlying bar graph recognition, and the bar graph factor was found to correlate significantly to the dot-subitizing factor but not to the dot-counting factor. Finally, VF asymmetry in the bar graph task correlated significantly to asymmetry in dot subitizing but not in dot counting, and the dot-subitizing and dot-counting asymmetries were independent of one another.

The recurring scallop-shaped outcome in RT suggests that bar graph recognition, like dot enumeration subitiz- 
ing, reflects a fast-operating cumulation process. However, whereas dot subitizing cumulates upward from a quantity of 0 , the quantification of bar graphs in Study 1 appears to cumulate upward from 0 , then from 4 , and then from 8 (see Figure 2).

Although the results may carry important implications for how the subitizing process is best characterized, it is important to keep in mind that the data analysis in Study 1 was post hoc, performed on a data set collected for other purposes. Furthermore, the factor-analytic outcome suggests that the design of the tasks was not optimal for isolating the three underlying processes. Specifically, whereas the bar graph task appeared to provide a pure measure of a single numerical process, the dot enumeration task involved two numerical processes, one used solely for the low range (1-3 dots), the other used solely for the high range ( $6-8$ dots), but with both processes used in mixture for the middle range ( $4-5$ dots).

To address these concerns, Study 2 was designed as a prospective study of enumeration and involved three separate tasks. The bar graph task was similar to that in Study 1, but two separate dot enumeration tasks were employed, one for the range of 1-4 dots and one for the range of 5-8 dots. It was hoped that by separating the dot stimuli into these ranges, purer measures of underlying numerical processes would be obtained. Also, because Study 1 showed correlations among VF asymmetries to be informative, the study was again run as a lateralization study involving bilateral displays of limited duration.

\section{STUDY 2}

\section{Method}

Participants. The sample initially comprised 95 young adults. However, the final sample comprised 46, after screening to remove (1) left-handed participants (as determined by the Edinburgh Handedness Inventory), (2) those with fewer than $60 \%$ correct responses in one or more of the three tasks, and (3) those with no correct responses for one or more numerical values in one or more of the tasks. The high rate of attrition was due mostly to poor performance on the task involving high numbers of dots; not surprisingly, counting is not very accurate for relatively high numbers of dots when display times are short.

Apparatus and Stimuli. Stimuli were presented in a white color on a dark background, using a 14-in. color monitor driven by an Apple iMac computer and SuperLab Pro software. Dot and bar graph stimuli were similar in form to those used in Study 1, although the transfer of the programs to a different computer system resulted in minor dimensional changes. Thus, a dot cluster subtended $4.9^{\circ} \times$ $5.9^{\circ}$ with an eccentricity of $3.3^{\circ}$, and a bar graph subtended $2.0^{\circ} \times$ $7.6^{\circ}$ with an eccentricity of $2.6^{\circ}$. Responses were collected on two keyboard keys centered in front of the participant, one located away from the other.

Procedure. The tasks used the same time line and responses as in Study 1, with the exception that the stimulus duration was increased from 100 to $180 \mathrm{msec}$ to make the tasks less difficult, especially the one using a high number of dots. Feedback consisted of "Correct!" or "Error" displayed on the screen. In all tasks, 24 practice trials were followed by 144 experimental trials, the latter split into two blocks of 72 with the opportunity for a brief rest in between. All participants received all three tasks, with task order and hand assignments counterbalanced across participants as evenly as possible given the sample size.

\section{Results}

Correct responses were analyzed as in Study 1.

RT data. The RT data are illustrated in Figure 4. An ANOVA like the one used in Study 1 was conducted, with the low-dots and high-dots data combined to provide results for the full numerical range of 1-8 dots. All three main effects were significant $\left[F(1,45)=12.33, M S_{\mathrm{e}}=\right.$ $18,904, p=.001$, for VF; $F(7,315)=118.73, M S_{\mathrm{e}}=$ $86,613, p<.001$, for number; and $F(1,45)=78.96$, $M S_{\mathrm{e}}=557,407, p<.001$, for task]. Strictly interpreted, the VF effect indicates that a general LVF advantage existed, which did not interact with task $[F(1,45)=0.41$, $\left.M S_{\mathrm{e}}=15,173\right]$. However, this outcome may be misleading since the dots data consisted of data from the separate low-dots and high-dots tasks combined, and Figure 4 suggests that the LVF advantage may occur only in the low-dots task. When the dots data were structured as a separate ANOVA, with low dots and high dots as separate levels of the task factor, there was in fact a significant VF $\times$ task interaction $\left[F(1,45)=7.56, M S_{\mathrm{e}}=3.47, p<.01\right]$. Separate ANOVAs then confirmed that an LVF advantage was found for the low-dots task $\left[F(1,45)=13.87, M S_{\mathrm{e}}=\right.$ $4,871, p=.001]$ but not for the high-dots task $[F(1,45)=$ $\left.0.52, M S_{\mathrm{e}}=40,254\right]$.

Returning to the larger analysis, the VF $\times$ number and number $\times$ task interactions were both significant $\left[F(7,315)=7.12, M S_{\mathrm{e}}=12,978, p<.001\right.$, and $F(7,315)=118.95, M S_{\mathrm{e}}=84,377, p<.001$, respectively]. However, these were subsumed by the significant interaction of $\mathrm{VF} \times$ number $\times$ task $[F(7,315)=3.45$, $\left.M S_{\mathrm{e}}=15,172, p=.001\right]$.

The three-way interaction was decomposed by examining the $\mathrm{VF} \times$ number interaction separately for each task. This was significant for bar graphs $[F(7,315)=12.29$, $\left.M S_{\mathrm{e}}=11,359, p<.001\right]$ but not for dots $[F(7,315)=$ $\left.0.31, M S_{\mathrm{e}}=16,791\right]$. As Figure 4 illustrates, for bar graphs, the LVF has a scalloped function, whereas RVF RTs are relatively flat over number values.

The interaction raises the possibility that different processes were used for LVF and RVF bar graphs. To further explore this possibility, a correlational analysis was

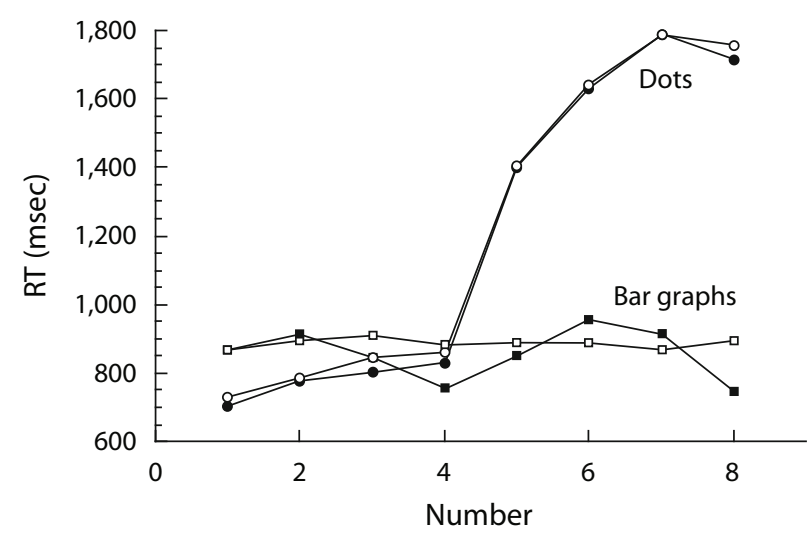

Figure 4. Response times from Study 2 . The closed symbols represent the left visual field; open symbols, the right visual field. 
undertaken in which mean RTs for LVF and RVF bar graphs were correlated to mean RTs for low dots and high dots. It was found that both LVF and RVF bar graph RTs correlated significantly more highly with low-dots RTs $[r(44)=+.67$ and +.62 , respectively $]$ than with highdots RTs $[r(44)=+.39$ and +.34 , respectively] $(z=$ $2.41, p<.02$, and $z=2.32, p<.05$ ). This outcome suggests that, the ANOVA interaction notwithstanding, the same process is used for LVF and RVF bar graphs, and it is also the same process used for enumerating dots in the low (1-4) range.

Further support for this conclusion comes from the similarity of the results from Studies 1 and 2 when the bar graph data are collapsed over VF. Factor analysis of the bar graph results was conducted by calculating mean RTs across VFs separately for each numerical value and subjecting the resulting eight values to principal-components analysis. Only one factor was extracted, with an eigenvalue of 6.70 (with the next eigenvalue at 0.33 ), which accounted for $84 \%$ of the variance. All factor matrix loadings ranged between .88 and .95 . These results are highly similar to those from Study 1.

Factor analysis of the dot enumeration data resulted in the extraction of two factors, with eigenvalues of 5.47 and 1.65 (the next having a value of 0.29 ), which accounted for $89 \%$ of the variance. Varimax rotation converged in three iterations, producing the values shown in Table 2. The results are virtually the same as those from Study 1, but with no ambiguity concerning the loadings of the 4and 5-dot values.

As in Study 1, factor scores were next calculated for the single bar graph factor and the two dot enumeration factors (again termed the subitizing factor and the counting factor), and correlations among them were assessed. The bar graph factor score was significantly correlated to the subitizing factor score $[r(44)=+.63, p<.001]$ but not to the counting factor score $[r(44)=+.21, p>.10]$. A similar analysis conducted on the VF difference scores showed that bar graph asymmetry correlated significantly with subitizing asymmetry $[r(44)=+.37, p<.02]$ but not with counting asymmetry $[r(44)=-.02]$. These results are again very similar to those of Study 1.

Percentage errors. The percentage error data are illustrated in Figure 5. Although noisier, the error data appear to reflect trends similar to those in the RT data, as they did in Study 1.

Table 2

Rotated Factor Matrix Loadings From Study 2, With All Loadings Greater Than .40 Shown

\begin{tabular}{ccc}
\multicolumn{3}{c}{ Loadings Greater Than .40 Shown } \\
\hline Variable & Factor 1 & Factor 2 \\
\hline 1 dot & & .93 \\
2 dots & & .91 \\
3 dots & & .90 \\
4 dots & .88 & .84 \\
5 dots & .92 & \\
6 dots & .92 & \\
7 dots & .93 & \\
8 dots & & \\
\hline
\end{tabular}

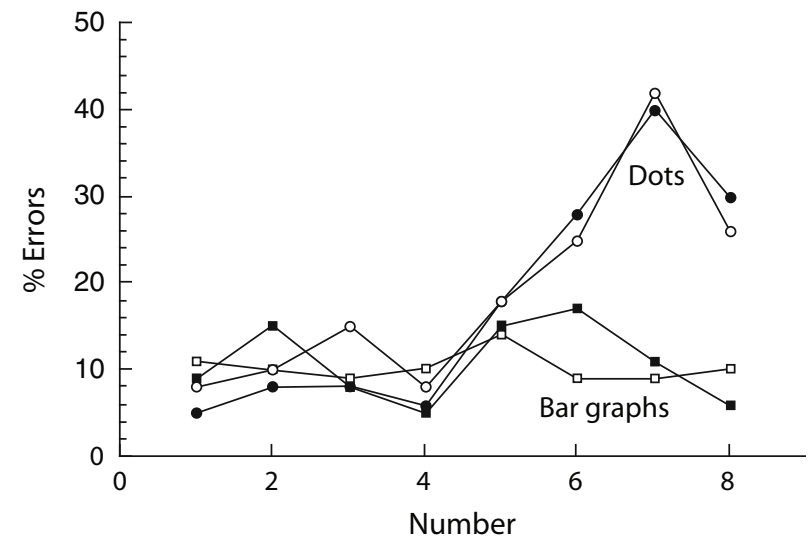

Figure 5. Percentage errors from Study 2. The closed symbols represent the left visual field; open symbols, the right visual field.

\section{Discussion}

Using a prospective design and separate tasks for low and high ranges of dots, Study 2 confirmed the major findings of Study 1 with some alteration of detail. Factor analysis revealed separate processes underlying the subitizing and counting ranges of dot enumeration and a single numerical process underlying bar graph recognition. In detail, the factor-analytic results differed from those of Study 1 in that the separate dot enumeration processes were completely disjoint, with no mixture of processes evident in the $4-5$ dot range. Thus, the use of separate tasks for bar graphs and each of the two dot ranges succeeded in producing a pure measure from each task, presumably because participants could consistently use the appropriate subitizing or counting process across all trials in a task.

The correlational results were also similar to those from Study 1. Both in overall RT and VF asymmetry, the process underlying bar graph recognition showed commonality with the dot-subitizing but not with the dot-counting process.

The forms of the functions relating numerical value to RT were a bit more complex than in Study 1, but in the end, they appear to yield similar conclusions. A low-slope, scallop-shaped function was again found for bar graphs, although it primarily described LVF trials. RVF trials produced a flat RT profile. Nevertheless, both LVF and RVF RTs correlated more highly with dot-subitizing RTs than with dot-counting RTs, reinforcing the conclusion that bar graph recognition and dot subitizing involve the same process, whereas dot counting involves a different process. In any case, the steep RT function for dot counting again sets that process apart from the processes underlying bar graph recognition and dot subitizing.

\section{GENERAL DISCUSSION}

The present research began with the observation that although dot enumeration and bar graph recognition have little formal similarity, they nevertheless can show evidence of involving a common numerical process, according to studies employing factor analysis of lateral differences, 
transfer of training, and time-sharing (Boles, 1991, 1997; Boles \& Law, 1998). The key to unraveling the mystery of what two seemingly unlike things have in common proves to be the multiple components that previous research found to underlie dot enumeration (see, e.g., Mandler \& Shebo, 1982; Trick \& Pylyshyn, 1993, 1994). The discovery of commonality between bar graph recognition and the enumeration of dots in the low or subitizing range affords a satisfying explanation of the observation that motivated the study. More importantly, the results seem to bear important implications about the nature of subitizing.

Across both studies, virtually all relevant results indicate that bar graph recognition and dot enumeration in the 1-4 dot range relied on the same subitizing process. Both tasks produced shallow RT slopes and unitary factors from factor analysis that positively correlated with one another, both failed to show correlations to the factor underlying dot enumeration within the counting range, both showed LVF asymmetries that positively correlated, and both asymmetries failed to correlate with countingrange asymmetry. The results therefore appear to indicate that subitizing underlies bar graph recognition, at least as instantiated in the type of bar graphs used here.

Closer examination of the function relating RT to number value provides insight into what it means to subitize when recognizing bar graphs. In Study 1, the results were consistent with a fast-incrementing process that started at value 0 , then restarted at values 4 and 8 . In each case, the starting value corresponded to a horizontal reference line placed on the bar graph against which values were to be judged. It seems apparent that in order to judge the odd or even value of the bar graphs in Study 1, participants simply began incrementing in whole numbers from whichever reference line was positioned immediately under the bar graph marker. Presumably, this involved imagining intervals corresponding to an increment of one and a summation of intervals until the target number was reached.

The form of the RT curve in Study 2 similarly suggests a fast, subitizing process, at least in the case of LVF trials. However, the results also indicate that incrementing from the horizontal reference lines 0,4 , and 8 was not obligatory. In this study, RTs were longest at the intermediate points between reference lines, specifically at 2 and 6 , and they lessened in either direction. The pattern strongly suggests that participants started at an intermediate point and incremented or decremented as needed to judge the odd or even value of the bar graph.

It is difficult to say why participants used different strategies in the two studies, but one possible explanation is that the participants in Study 1 were almost exclusively from a technological university at which students have considerable experience in reading graphs, whereas Study 2 drew participants from a general-studies university. In any case, the variation in results between studies seems less important than the fact that a scalloped RT function was found in at least some conditions of both.

\section{What Is Subitizing?}

Although our inquiry began with the observation that dot enumeration and bar graph recognition can have a perceptual process in common, its significance lies in apparent implications for characterizing the process held in common, which we take to be subitizing.

An initial, though not surprising, conclusion is that subitizing is much faster than counting, as indicated by the much steeper slopes of the RT function for the 5-8 dot range. Nevertheless, subitizing seems to share one characteristic with counting - namely, an increase (albeit a small one) in RTs as numerical values increase within its range. A second conclusion about subitizing is that under appropriate circumstances, it can be used for both dot enumeration and bar graph recognition. Third, it is presumably not language-like, in that an LVF (right-hemisphere) advantage was found for both dot subitizing and bar graph recognition. Fourth, Study 2 indicated that the subitizing process can operate bidirectionally, using either increments or decrements.

We believe that these characteristics of subitizing, paired with the nature of the bar graph stimuli, add up to the conclusion that the subitizing process is a nonverbal one operating on analog quantities having spatial extent. In the case of dot patterns, the analog quantities are the dots, but in the case of bar graphs, they are imaginary intervals or steps above or below reference lines. Nevertheless, the same process operates in both cases, and it seems appropriate to refer to it as a nonverbal process of fast counting or, in the case of decrements, a nonverbal process of fast subtraction. Presumably, the use of this same process for both bar graphs and low-range dot enumeration trials accounts for common factor loadings (Boles, 1991), transfer of training from one task to the other (Boles, 1997), and selective interference in time-sharing experiments (Boles \& Law, 1998).

It is illuminating to evaluate existing models of subitizing in light of the present results. Bar graphs varying in value from 1-8 do not produce patterns such as lines or triangles, thus disfavoring models based on canonical patterns or similarity of form (Logan \& Zbrodoff, 2003; Mandler \& Shebo, 1982). Each value of a bar graph depends on a single location of an indicator plotted against fixed reference lines; thus, models based on Weber's law, limits in short-term memory, or the number of tagged item locations seem similarly disfavored (Averbach, 1963; Trick \& Pylyshyn, 1993, 1994). In particular, it is unclear how such models can account for scalloped RT functions given a fixed (constant) total of reference lines plus an indicator location.

In contrast, the preverbal fast-counting model of Gallistel and Gelman (1992) seems quite consistent with the data from the present studies and is very similar to what we propose, differing only in that their model makes no allowance for counting decrements.

Finally, we should note that the somewhat artificial nature of the bar graphs, with horizontal reference markers at the 0,4 , and 8 levels, appears to have induced the use of the subitizing process. This was fortuitous in that having induced the process, the nature of the bar graphs speaks to the nature of the subitizing process. Whether other bar graph designs or presentation conditions would do the same is largely irrelevant to our conclusions. 


\section{Lateralization}

It seems appropriate to revisit the starting point of this inquiry by considering lateral differences in numerical processes. The most consistent lateralization finding over both studies was an LVF (right-hemisphere) advantage in processing produced by the subitizing process, whether in bar graphs or in the low range of dot enumeration. The outcome is consistent with our conception of subitizing as a nonverbal process that produces fast counting or subtraction of analog quantities, a spatial operation that sensibly involves the right hemisphere (Boles, 2002).

In contrast, the results of Study 2 indicate that when enumeration in the high range of 5-8 dots is made a separate, isolated task, an LVF advantage is no longer found. The outcome indicates a shift away from right-hemisphere processing when subitizing is no longer used, but it stops short of establishing that the left hemisphere is emphasized. Interestingly, the lack of a VF difference is largely consistent with the previously cited PET study by Sathian et al. (1999), who likewise failed to find the preferential left-hemisphere involvement that would seem to be predicted by a verbal counting process. At least a couple of possibilities exist for why greater left-hemisphere involvement is not found. First, counting may itself be composed of a number of subprocesses, including the deployment of spatial attention and maintenance of a running total (Trick \& Pylyshyn, 1994), as well as verbal labeling. The mixture of subprocesses may therefore be such that neither hemisphere has a particular edge. A second possibility is that counting falls within an atypical behavioral domain in which the two hemispheres both have access to verbal methods of differing kinds. Van Lancker and Cummings (1999) indicated that when the left-hemisphere propositional speech system is damaged, producing aphasia, automatized speech is often spared. Such speech includes coprolalia and, significantly, counting up to some small number - for example, from 1 to 10. Presumably, it is the undamaged right hemisphere that is responsible for such speech. According to this explanation, both propositional and automatic modes of speech are possible in counting, affording an advantage to neither hemisphere.

The results of the two studies give very little indication of an estimation process that operates on a large number of dots. Judging from the factor-analytic results, it appears that when patterns of 6-8 dots were included as part of the high range of 4-8 dots, they were recognized using the slow counting process. It is possible that an end effect prevented the use of an estimation process in our studies, with participants judging that 8 dots had been presented as soon as they counted past 7 dots.

\section{Could the Results Be Artifacts of Error Rates or General Difficulty?}

It is worth pointing out that the systematic and converging nature of the evidence argues against two possible artifactual accounts of the results. One such account is based on the fact that the high range of dots produced a high rate of errors compared with the error rates produced by bar graphs and the low range of dots. The apparent instability of the high-dots data might then result in high error variance that prevents the data from correlating with data from either low dots or bar graphs, whose more stable data allow relationships between them to emerge. Such an account, however, has considerable difficulty explaining the near-perfect factor structure among high-dots stimuli (Tables 1 and 2), which indicates that the data are far from unstable. Indeed, further analysis shows the raw correlations $(r)$ among the individual 5- to 8-dot stimuli to be substantial in both studies, ranging from .75-.88 in Study 1 and from .81-.92 in Study 2, and quite comparable to correlations among both the 1- to 4-dot stimuli (.53-.79 in Study 1 and .74-.89 in Study 2) and the full 1-8 range of bar graphs (.61-.83 in Study 1 and .74-.91 in Study 2).

Similar considerations apply to an attempt to explain the results in terms of general difficulty - based, for example, on the supposition that less difficult stimuli of any type might intercorrelate more highly than more difficult stimuli. Besides being hard to rationalize on a theoretical basis, such an explanation has difficulty accounting for the bar graph results of Study 1, in which the easy 1, 4, 5, and 8 values (i.e., fast and accurate; see Figures 2 and 3 ) produced roughly equivalent correlations among themselves, ranging from .71-.80, and to the difficult $2,3,6$, and 7 values (i.e., markedly slower and error prone; see Figures 2 and 3), ranging from .61-.74. In the factor analysis, these correlations were all large enough to result in a single factor. In contrast, the individual ranges of 1- to 3-dot stimuli and 6- to 8-dot stimuli (avoiding the mixture values of 4- and 5-dot stimuli) showed markedly smaller correlations across the subsets, ranging from .23-.46. In the factor analysis, these smaller correlations were insufficient to support common factor loadings. Thus, easy and difficult bar graph stimuli involve the same process, whereas easy and difficult dot stimuli do not, considerably weakening the general difficulty explanation. Also, because this explanation is not tied to hemispheric differences, it provides no ready account for the correlations observed in VF asymmetry that support commonality between the low range of dots and bar graphs, but not between either of them and the high range of dots. In conclusion, we see no reasonable basis for supposing that the results are artifacts either of error rates or of general difficulty.

\section{AUTHOR NOTE}

We thank Lindsey Adair for helping with participant recruitment and Jenny Abernethy, Chris Fullerton, Jason Perdelwitz, and Matt Steimle for running participants. Correspondence concerning this article should be addressed to D. B. Boles, Department of Psychology, P.O. Box 870348, Tuscaloosa, AL 35405 (e-mail: dboles@bama.ua.edu).

\section{REFERENCES}

Averbach, E. (1963). The span of apprehension as a function of exposure duration. Journal of Verbal Learning \& Verbal Behavior, 2, 60-64.

Basak, C., \& Verhaeghen, P. (2003). Subitizing speed, subitizing range, counting speed, the Stroop effect, and aging: Capacity differences and speed equivalence. Psychology \& Aging, 18, 240-249.

Boles, D. B. (1983). Hemispheric interaction in visual field asymmetry. Cortex, 19, 99-113.

Boles, D. B. (1987). Reaction time asymmetry through bilateral versus unilateral stimulus presentation. Brain \& Cognition, 6, 321-333. 
Boles, D. B. (1990). What bilateral displays do. Brain \& Cognition, 12, 205-228.

BoLes, D. B. (1991). Factor analysis and the cerebral hemispheres: Pilot study and parietal functions. Neuropsychologia, 29, 59-91.

Boles, D. B. (1992). Factor analysis and the cerebral hemispheres: Temporal, occipital and frontal functions. Neuropsychologia, 30, 963-988.

Boles, D. B. (1995). Parameters of the bilateral effect. In F. L. Kitterle (Ed.), Hemispheric communication: Mechanisms and models (pp. 211-230). Hillsdale, NJ: Erlbaum.

BoLES, D. B. (1996). Factor analysis and the cerebral hemispheres: "Unlocalized" functions. Neuropsychologia, 34, 723-736.

Boles, D. B. (1997). Multiple resource contributions to training. Proceedings of the Human Factors Society, 41, 1176-1179.

BOLES, D. B. (1998). Relationships among multiple task asymmetries: II. A large-sample factor analysis. Brain \& Cognition, 36, 268-289.

Boles, D. B. (2002). Lateralized spatial processes and their lexical implications. Neuropsychologia, 40, 2125-2135.

Boles, D. B., \& LAW, M. B. (1998). A simultaneous task comparison of differentiated and undifferentiated hemispheric resource theories. Journal of Experimental Psychology: Human Perception \& Performance, 24, 204-215.

ChI, M. T. H., \& KLahr, D. (1975). Span and rate of apprehension in children and adults. Journal of Experimental Child Psychology, 19, 434-439.

Dehaene, S., \& Cohen, L. (1994). Dissociable mechanisms of subitizing and counting: Neuropsychological evidence from simultanagnosic patients. Journal of Experimental Psychology: Human Perception \& Performance, 20, 958-975.

Fink, G. R., Marshall, J. C., Gurd, J., Weiss, P. H., Zafiris, O., Shah, N. J., \& Zilles, K. (2001). Deriving numerosity and shape from identical visual displays. NeuroImage, 13, 46-55.

Gallistel, C. R., \& Gelman, R. (1992). Preverbal and verbal counting and computation. Cognition, 44, 43-74.

GoRsuch, R. L. (1983). Factor analysis. Hillsdale, NJ: Erlbaum.

Kaufman, E. L., Lord, M. W., Reese, T. W., \& Volkmann, J. (1949). The discrimination of visual number. American Journal of Psychology, 62, 498-525.

Logan, G. D., \& ZBrodoff, N. J. (2003). Subitizing and similarity: Toward a pattern-matching theory of enumeration. Psychonomic Bulletin \& Review, 10, 676-682.

NAN, Y., KNÖsche, T. R., \& LUO, Y.-J. (2006). Counting in everday life: Discrimination and enumeration. Neuropsychologia, 44, 1103-1113.

Mandler, G., \& Shebo, B. J. (1982). Subitizing: An analysis of its component processes. Journal of Experimental Psychology: General, 111, 1-22.

OldFIELD, R. C. (1971). The assessment and analysis of handedness: The Edinburgh inventory. Neuropsychologia, 9, 97-113.

OlK, B., \& HaRTJE, W. (2001). The bilateral effect: Callosal inhibition or intrahemispheric competition? Brain \& Cognition, 45, 317-324.

OsGood, G. (1988). Generalizing the Apple-Psych system. Behavior Research Methods, Instruments, \& Computers, 20, 155-157.

Oyama, T., Kikuchi, T., \& Ichinara, S. (1981). Span of attention, backward masking, and reaction time. Perception \& Psychophysics, 29, 106-112.

RAYMAN, J., \& ZAIDEL, E. (1991). Rhyming and the right hemisphere. Brain \& Language, 40, 89-105.

Sathian, K., Simon, T. J., Peterson, S., Patel, G. A., Hoffman, J. M., \& Grafton, S. T. (1999). Neural evidence linking visual object enumeration and attention. Journal of Cognitive Neuroscience, 11, 36-51.

Svenson, O., \& SJöBerg, K. (1978). Subitizing and counting processes in young children. Scandinavian Journal of Psychology, 19, 247-250.

Trick, L. M., \& Pylyshyn, Z. W. (1993). What enumeration studies can show us about spatial attention: Evidence for limited capacity preattentive processing. Journal of Experimental Psychology: Human Perception \& Performance, 19, 331-351.

TRICK, L. M., \& PYLYShYN, Z. W. (1994). Why are small and large numbers enumerated differently? A limited-capacity preattentive stage in vision. Psychological Review, 101, 80-102.

Tuholski, S. W., Engle, R. W., \& Baylis, G. C. (2001). Individual differences in working memory capacity and enumeration. Memory \& Cognition, 29, 484-492.

van LANCKer, D., \& Cummings, J. L. (1999). Expletives: Neurolinguistic and neurobehavioral perspectives on swearing. Brain Research Reviews, 31, 83-104.

van Oeffelen, M. P., \& Vos, P. G. (1982). A probabilistic model for the discrimination of visual number. Perception \& Psychophysics, 32, 163-170.

WATSON, D. G., \& MAYLOR, E. A. (2006). Effects of color heterogeneity on subitization. Perception \& Psychophysics, 68, 319-326.

Wender, K. F., \& Rothkegel, R. (2000). Subitizing and its subprocesses. Psychological Research, 64, 81-92.

(Manuscript received February 7, 2006; revision accepted for publication January 3, 2007). 